\title{
A Binomial Coefficient Identity Associated with Beukers' Conjecture on Apéry numbers
}

\author{
CHU Wenchang* \\ College of Advanced Science and Technology \\ Dalian University of Technology \\ Dalian 116024, P. R. China \\ chu. wenchang@unile.it
}

Submitted: Oct 2, 2004; Accepted: Nov 4, 2004; Published: Nov 22, 2004

Mathematics Subject Classifications: 05A19, 11P83

\begin{abstract}
By means of partial fraction decomposition, an algebraic identity on rational function is established. Its limiting case leads us to a harmonic number identity, which in turn has been shown to imply Beukers' conjecture on the congruence of Apéry numbers.
\end{abstract}

Throughout this work, we shall use the following standard notation:

$\left.\begin{array}{lccc}\text { Harmonic numbers } & H_{0}=0 & \text { and } & H_{n}=\sum_{k=1}^{n} 1 / k \\ \text { Shifted factorials } & (x)_{0}=1 & \text { and } & (x)_{n}=\prod_{k=0}^{n-1}(x+k)\end{array}\right\}$ for $n=1,2, \cdots$.

For a natural number $n$, let $A(n)$ be Apéry number defined by binomial sum

$$
A(n):=\sum_{k=0}^{n}\left(\begin{array}{l}
n \\
k
\end{array}\right)^{2}\left(\begin{array}{c}
n+k \\
k
\end{array}\right)^{2}
$$

and $\alpha(n)$ determined by the formal power series expansion

$$
\sum_{m=1}^{\infty} \alpha(m) q^{m}:=q \prod_{n=1}^{\infty}\left(1-q^{2 n}\right)^{4}\left(1-q^{4 n}\right)^{4}=q-4 q^{3}-2 q^{5}+24 q^{7}+\cdots
$$

Beukers' conjecture [3] asserts that if $p$ is an odd prime, then there holds the following congruence (cf. [1, Theorem 7])

$$
A\left(\frac{p-1}{2}\right) \equiv \alpha(p) \quad\left(\bmod p^{2}\right)
$$

\footnotetext{
${ }^{*}$ The work carried out during the summer visit to Dalian University of Technology (2004).
} 
Recently, Ahlgren and Ono [1] have shown that this conjecture is implied by the following beautiful binomial identity

$$
\sum_{k=1}^{n}\left(\begin{array}{l}
n \\
k
\end{array}\right)^{2}\left(\begin{array}{c}
n+k \\
k
\end{array}\right)^{2}\left\{1+2 k H_{n+k}+2 k H_{n-k}-4 k H_{k}\right\}=0
$$

which has been confirmed successfully by the WZ method in [2].

The purpose of this note is to present a new and classical proof of this binomialharmonic number identity, which will be accomplished by the following general algebraic identity.

Theorem. Let $x$ be an indeterminate and $n$ a natural number. There holds

$$
\frac{x(1-x)_{n}^{2}}{(x)_{n+1}^{2}}=\frac{1}{x}+\sum_{k=1}^{n}\left(\begin{array}{c}
n \\
k
\end{array}\right)^{2}\left(\begin{array}{c}
n+k \\
k
\end{array}\right)^{2}\left\{\frac{-k}{(x+k)^{2}}+\frac{1+2 k H_{n+k}+2 k H_{n-k}-4 k H_{k}}{x+k}\right\} .
$$

The binomial-harmonic number identity (1) is the limiting case of this theorem. In fact, multiplying by $x$ across equation (2) and then letting $x \rightarrow+\infty$, we recover immediately identity (1).

Proof of the Theorem. By means of the standard partial fraction decomposition, we can formally write

$$
f(x):=\frac{x(1-x)_{n}^{2}}{(x)_{n+1}^{2}}=\frac{A}{x}+\sum_{k=1}^{n}\left\{\frac{B_{k}}{(x+k)^{2}}+\frac{C_{k}}{x+k}\right\}
$$

where the coefficients $A$ and $\left\{B_{k}, C_{k}\right\}$ remain to be determined.

First, the coefficients $A$ and $\left\{B_{k}\right\}$ are easily computed:

$$
\begin{aligned}
A & =\lim _{x \rightarrow 0} x f(x)=\lim _{x \rightarrow 0} \frac{(1-x)_{n}^{2}}{(1+x)_{n}^{2}}=1 ; \\
B_{k} & =\lim _{x \rightarrow-k}(x+k)^{2} f(x)=\lim _{x \rightarrow-k} \frac{x(1-x)_{n}^{2}}{(x)_{k}^{2}(1+x+k)_{n-k}^{2}} \\
& =\frac{-k(1+k)_{n}^{2}}{(-k)_{k}^{2}(1)_{n-k}^{2}}=-k\left(\begin{array}{c}
n \\
k
\end{array}\right)^{2}\left(\begin{array}{c}
n+k \\
k
\end{array}\right)^{2} .
\end{aligned}
$$

Applying the L'Hôspital rule, we determine further the coefficients $\left\{C_{k}\right\}$ as follows:

$$
\begin{aligned}
C_{k} & =\lim _{x \rightarrow-k}(x+k)\left\{f(x)-\frac{B_{k}}{(x+k)^{2}}\right\}=\lim _{x \rightarrow-k} \frac{(x+k)^{2} f(x)-B_{k}}{x+k} \\
& =\lim _{x \rightarrow-k} \frac{d}{d x}\left\{(x+k)^{2} f(x)-B_{k}\right\}=\lim _{x \rightarrow-k} \frac{d}{d x} \frac{x(1-x)_{n}^{2}}{(x)_{k}^{2}(1+x+k)_{n-k}^{2}} \\
& =\lim _{x \rightarrow-k} \frac{(1-x)_{n}^{2}}{(x)_{k}^{2}(1+x+k)_{n-k}^{2}}\left\{1-\sum_{i=1}^{n} \frac{2 x}{i-x}-\sum_{\substack{j=0 \\
j \neq k}}^{n} \frac{2 x}{x+j}\right\} \\
& =\left(\begin{array}{c}
n \\
k
\end{array}\right)^{2}\left(\begin{array}{c}
n+k \\
k
\end{array}\right)^{2}\left\{1+2 k H_{n+k}+2 k H_{n-k}-4 k H_{k}\right\} .
\end{aligned}
$$

This completes the proof of the Theorem. 


\section{References}

[1] S. Ahlgren - K. Ono, A Gaussian hypergeometric series evaluation and Apéry number congruences, J. Reine Angew. Math. 518 (2000), 187-212.

[2] S. Ahlgren - S. B. Ekhad - K. Ono - D. Zeilberger, A binomial coefficient identity associated to a conjecture of Beukers, The Electronic J. Combinatorics 5 (1998), \#R10.

[3] F. Beukers, Another congruence for Apéry numbers, J. Number Theory 25 (1987), 201-210.

Current Address:

Dipartimento di Matematica

Università degli Studi di Lecce

Lecce-Arnesano P. O. Box 193

73100 Lecce, ITALIA

Email chu.wenchang@unile.it 